\title{
Analysis of the Chaotic Phenomena in Securities Business of China
}

\author{
Chong $\mathrm{Fu}^{1}$, Su-Ju Li ${ }^{2}$, Hai $\mathrm{Yu}^{1}$, and Wei-Yong $\mathrm{Zhu}^{1}$ \\ ${ }^{1}$ School of Information Science and Engineering, Northeastern University, \\ 110004 Shenyang, China \\ \{fu_chong, yu_hai, zhu_weiyong\} @sohu.com \\ ${ }^{2}$ College of Foreign Studies, Northeastern University, \\ 110004 Shenyang, China
}

\begin{abstract}
The tendency of stock price in securities business based on the Shenzhen stock composite index was studied by using chaotic dynamics theory. The fluctuation of stock price was proved to be a kind of chaotic process of inner random. The dynamic model of Shenzhen stock composite index was established both by the restructure of phase space of the data and by the analysis of the Poincaré section and Lyapunov exponent of the data. The chaotic evolvement process of this model was analyzed in detail. This provides a new method for the investigation of modern financial system by the use of chaotic theory.
\end{abstract}

\section{Introduction}

In the early 1900s, French mathematician Louis Bacheiler proposed a model describing the rules of price fluctuation, which became the foundation of modern financial securities theory. This model assumes that the price fluctuation obeys normal distribution and points out that the changes of the price are independent in statistics and follows the "Random Walk" which accords with the bell-like curve. According to his theory, the price should fluctuate slightly in a small range, the violent shakeout should seldom happen, but it exceeds people's expectation greatly. This indicates that current securities theory cannot give a correct explanation to the large fluctuation of finance system.

In 1990s, Professor B.B.Mandelbrot, the academician of American Academy and founder of fractal theory, proposed a new multi fractal model to describe the fluctuation of the securities market. It accurately describes the relationship of the up and down fluctuation, revealing the essential of the frequent violent fluctuation of securities market, providing a determinative analysis method for the study of unpredictable financial system [1]. At present, most of the existing financial models are based on "Random Walk" theory, few scholars set up financial model on the basis of chaos theory. In this paper, the chaotic dynamics theory was used to analyze the chaotic property of securities market and a chaotic iteration model was established to further study the evolvement process of the complicated financial system. 


\section{Power Spectrum Analysis of Trading Sequence}

The tendency chart of composite index in Shenzhen securities between $8-17^{\text {th }}-1992$ and $8-31^{\text {th }}-2004$ is shown in Fig. 1. The sampling interval is based on day.

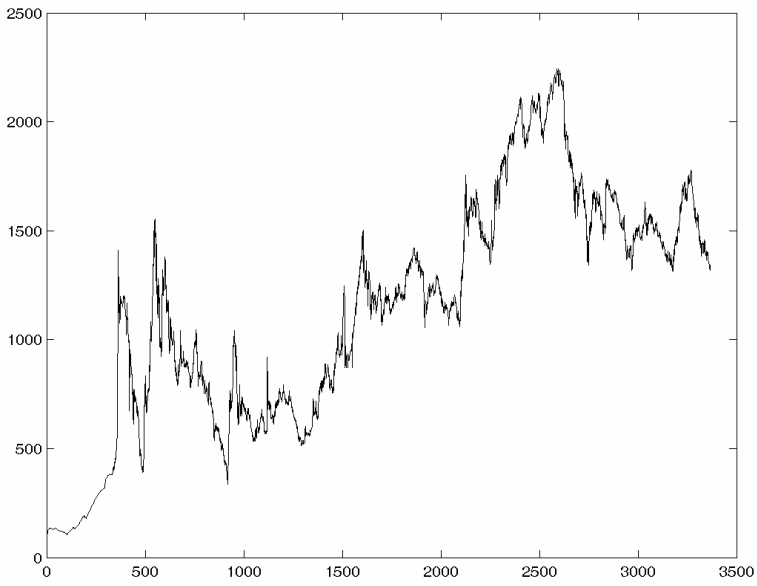

Fig. 1. Tendency chart of composite index of Shenzhen securities between $8-17^{\text {th }}-1992$ and $8-31^{\text {th }}-2004$

Power spectrum analysis is often used to identify the chaos behavior of a system. For sequence in Fig. 1, we do discrete Fourier transform, calculate its Fourier coefficient

$$
F(u)=\frac{1}{N} \sum_{i=0}^{N-1} x_{i} \exp \{-j 2 \pi u x / N\}
$$

The result is shown in Fig. 2. The power spectrum of the chaotic system shows a peak on a broadband, which is different from periodic movements or Gaussian noise. From Fig. 2 we can see, the power changes continuously in frequency domain, it shows a downward trend in the form of negative exponent. Some irregular peaks are on the background, demonstrating period 3 phenomenon.

\section{Phase Space Reconstruction and Poincaré Section}

The chaotic attractor is the fundamental reason of some random movements. The first step of analyzing the chaotic property of a time sequence is reconstructing the attractor based on the experiment data. For time sequence $x_{0}, x_{1}, x_{2}, \ldots x_{N-1}$ ( $N$ must be large enough), we can get other $d-1$ dimension data by using the time delay method. The coordinate axes are respectively $x(t), x(t+m), x(t+2 m), \ldots, x(t+(d-1) m), m$ is the selected delay number. All the data determine a point in a multi dimension state space, then we can use other methods to examine whether it has a chaotic attractor. 


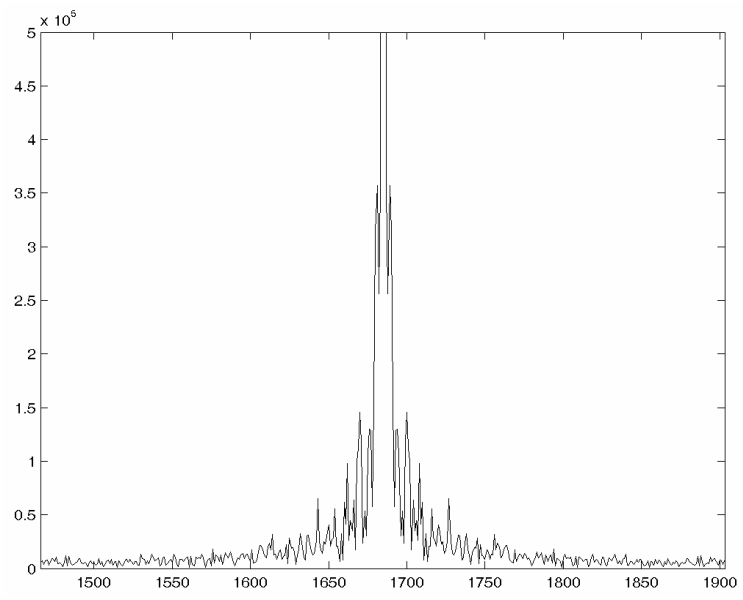

Fig. 2. Local enlarged power spectrum of Shenzhen securities composite index

The transection of attractors in phase space is Poincaré section. For a discrete time sequence in phase space, $X(t)$ presents current state, while $X(t+m)$ the state after delaying $m, X$ a $d$ dimension vector, the state transformations in $d$ dimension space is:

$$
(x(t+m), x(t), \ldots, x(t-(d-2) m))=F_{m}(x(t), x(t-m), \ldots, x(t-(d-1) m)) .
$$

The Poincaré section of Shenzhen securities composite index is shown in Fig. 3, the distribution of the points in the figure is very regular, the dynamic model is established as $[2,3]$ :

$$
X_{t+1}=r X_{t} \exp \left(-0.00034\left(1-X_{t}\right)^{2}\right) .
$$

$r$ is a random variable, $r \sim N(0.978,11.21)$.

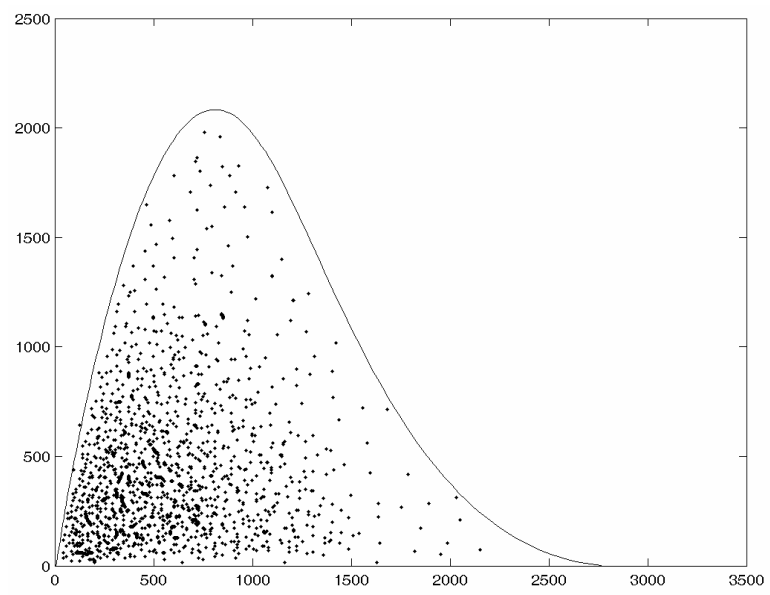

Fig. 3. Poincaré section and iterative model of Shenzhen securities composite index 


\section{Dynamical Characteristic Analysis of the Model}

The bifurcation diagram of dynamic model defined by Eq. 3 is shown in Fig. 4 [4].

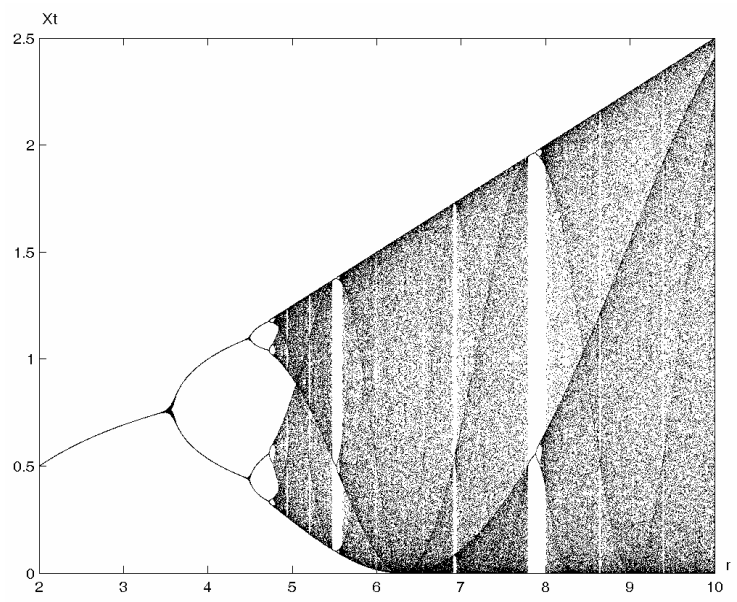

Fig. 4. Bifurcation diagram of the dynamical model defined by Eq. 3

When control parameter $r>4.716$, the system will come into chaos process. The evolvement of this system is a chaotic process, which contains periodic orbit, semiperiodic orbit, random orbit and chaos orbit.

\section{Conclusion}

This paper proves that the fluctuation of stock price is a mixed process, including periodic process and a topological invariance chaotic process. It provides a new idea and method of using the chaotic theory to study the fluctuation, inner principles and tendency of securities business.

\section{References}

1. Mandelbrot, B.B.: Fractals and Scaling in Finance. Springer-Verlag, Berlin Heidelberg New York (1997)

2. Liu, X.D., Zhu W.Y., Wang G.D.: Analysis of Chaotic Behavior of the Six-roll UC Mill and its OGY Control. Journal of Northeastern University, Vol. 19. (1997) 591-594

3. Ruelle, D., Takens, F.: On the Nature of Turbulence. Common Mathematics Physics, Vol. 20 (1971) 167-192

4. Feigenbaum, M.J.: Quantitative Universality for a Class of Nonlinear Transformations. J Statistic Phys, Vol. 19 (1978) 25-52 